\title{
Autoevaluación diagnóstica en enfermería
}

\section{Diagnostic self-assessment in nursing}

\section{Autoavaliação diagnóstica em enfermagem}

\author{
G. González-Juárez \\ ORCID: 0000-0003-4998-3653 \\ Recibido: 17 enero 2018 \\ Aceptado: 16 octubre 2018
}

Coordinación de Investigación. Escuela Nacional de Enfermería y Obstetricia, Universidad Nacional Autónoma de México, Ciudad de México, México

\section{Resumen}

Introducción: La evaluación educativa en enfermería que se realiza en la ENEO, ha representado múltiples esfuerzos de proyectos institucionales de carácter formativo. A más de dos décadas de experiencia, se requiere realizar un diagnóstico que permita identificar fortalezas y debilidades en tres componentes: planes de estudios, docencia y evaluación de los alumnos por exámenes.

Objetivo: Implementar la autoevaluación colegiada para identificar las fortalezas y debilidades de planes de estudio, docencia y exámenes, para valorar el aprovechamiento escolar de los alumnos del sistema escolarizado.

Método: El método fue exploratorio, con muestreo no probabilístico. Participaron 27 docentes en total, 25 mujeres y dos hombres, todos profesores de tiempo completo durante 14 sesiones totales, cuatro para cada uno de los grupos y sus objetos de evaluación y dos sesiones plenarias, una al inicio y otra al final. Se diseñó la autoevaluación estructurada de acuerdo con el Modelo Contexto, Insumo, Proceso y Producto (CIPP), para cada objeto de evaluación (plan de estudios, docencia y exámenes), se conformaron tres grupos durante cuatro sesiones de dos horas cada una y dos plenarias.

Resultados: En los tres componentes, el mayor promedio se ubica en las dificultades de los resultados institucionales y la mayor fortaleza se ubicó para planes de estudios, las debilidades se orientaron al bajo impacto de los exámenes.

Conclusiones: La evaluación educativa en enfermería sigue un modelo unilateral que limita la calidad y la toma de decisiones; para la mejora de las licenciaturas se requiere la presencia de otros actores a fin de fortalecerlas.

Palabras clave: Evaluación; evaluación curricular; evaluación de programas; métodos de evaluación; México. 


\section{Abstract}

Introduction: The assessment of nursing education at the National School of Nursing and Obstetrics has reflected diverse efforts from institutional projects. After two decades of experiences, it becomes necessary to carry out a diagnosis which can identify strengths and weaknesses related to three major components: study plans, teaching, and evaluation of students by means of exams.

Objective: To implement a collegiate self-assessment to identify strengths and weaknesses related to the study plans, the teaching process, and the given exams, to estimate the school performance of students within the in-situ system.

Method: This is an exploratory study with non-probabilistic sampling. There were 27 participants, 25 female and 2 male, all full-time professors, 4 for each one of the groups and its evaluation objects, and 2 plenary sessions, one at the beginning and the other at the end. A structured self-assessment was designed following the Context, Supply, Process and Product Model for each object of assessment (study plan, teaching, and exams).

Results: In the three components, the highest average concerns the difficulties of the institutional results, while the greatest strength were on the study plans, and the weaknesses were on the low impact of the exams.

Conclusions: Education assessment in nursing follows a unilateral model which limits the quality and decision making. In order to improve the undergraduate programs, it is necessary to include other corresponding actors and stakeholders.

Keywords: Assessment; curriculum assessment; program assessment; evaluation studies as topic; Mexico.

\section{Resumo}

Introdução: A avaliação educacional em enfermagem que é realizada na ENEO, tem representado múltiplos esforços de projetos institucionais de natureza formativa. Com mais de duas décadas de experiência, um diagnóstico é necessário para identificar pontos fortes e fracos em três componentes: currículos, ensino e avaliação dos alunos por meio de exames.

Objetivo: Implementar a autoavaliação colegiada para identificar os pontos fortes e fracos dos planos de estudo, ensino e exames, para avaliar o desempenho escolar dos alunos no sistema escolar.

Método: O método foi exploratório, com amostragem não probabilística. Um total de 27 professores participaram, 25 mulheres e dois homens, todos professores em tempo integral para 14 sessões no total, quatro para cada um dos grupos e seus objetos de avaliação e duas sessões plenárias, uma no início e uma no final. A auto-avaliação estruturada foi elaborada de acordo com o Contexto, Insumo, Processo e Modelo de Produto (CIPP), para cada objeto de avaliação (programa, ensino e exames), três grupos foram formados durante quatro sessões de duas horas cada e dois plenárias.

Resultados: Nos três componentes, a maior média está localizada nas dificuldades dos resultados institucionais e a maior força foi colocada nos planos de estudo, as fragilidades foram orientadas para o baixo impacto dos exames.

Conclusões: A avaliação educacional em enfermagem segue um modelo unilateral que limita qualidade e tomada de decisão; para a melhoria dos graus, é necessária a presença de outros atores para fortalecê-los. Palavras chave: Avaliação; avaliação curricular; avaliação de programas; métodos de avaliação; México.

\section{Introducción}

Las Instituciones educativas del siglo XXI, se constituyen como espacios capaces de hacer frente a las nuevas demandas de las sociedades multiculturales y respetuosas de la diversidad. Se organizan en comunidades de aprendizaje con expectativas de éxito respecto al potencial de los estudiantes; con plena conciencia de que todas las personas que la integran enseñan y aprenden, simultáneamente. La gestión del conocimiento se orienta en la democracia para rendir cuentas del cumplimiento de metas que la 
sociedad les demanda, la evaluación es un espacio privilegiado en la cadena de aprendizajes que potencia la reflexión y la toma de decisiones para reorientar los procesos de enseñanza y de aprendizaje ${ }^{1}$.

La rendición de cuentas se ha instrumentado a través de la evaluación educativa que realizan las Instituciones de Educación Superior (IES), tanto interna como externa, como pieza fundamental para aspirar al compromiso social, al empoderamiento y al trabajo colaborativo en las comunidades educativas ${ }^{2}$. Contribuye a la apropiación de una cultura de evaluación congruente con la misión y visión institucionales, pero encauzadas en la responsabilidad social de la Universidad ${ }^{3}$.

La literatura actual sobre evaluación educativa en educación superior enfatiza la importancia de la colaboración y la innovación de los actores educativos, para conformar comunidades de aprendizaje y alcanzar metas de calidad orientadas socialmente. La calidad convoca a diversos actores, momentos y enfoques para realizarla. En México, el Instituto Nacional de la Evaluación de la Educación, ha definido un esquema de criterios e indicadores de calidad que han sido, también enriquecidos con los grupos de enfoque convocados por los organismos evaluadores ${ }^{4}$.

No obstante, los avances que han permitido la presencia de la evaluación educativa en las IES, han sido más de forma que de fondo para orientar el cambio de las prácticas en torno al curriculum. El uso de las evaluaciones y la utilización de sus resultados ha sido un tema de atención en tres aspectos ${ }^{5}$ :

1). Qué tipo de uso es posible:

- Orientar las reformas de los programas (uso instrumental).

- Cambiar las ideas de las personas sobre el programa (uso conceptual).

- Movilizar apoyos a una posición como instrumento de persuasión.

- Efectos en otras instituciones más allá del programa, a través de la acumulación y difusión del conocimiento.

2). Qué es lo que se utiliza:

- Los resultados de la evaluación y las recomendaciones derivadas de ellos.

- Las ideas y generalizaciones derivadas de la evaluación.

- La evaluación en sí, para justificar acción o inacción.

3). Quiénes utilizan las evaluaciones:

- Los implicados directamente (directores, trabajadores del programa).

- Los clientes y usuarios (a quienes van dirigidos los programas).

- El público en general, los ciudadanos, que tienen derecho a la información ${ }^{6}$.

Entre los aspectos resaltados en este estudio, está el hecho de que la evaluación es una práctica orientada a la utilización de sus resultados y a la toma de decisiones, no así a las conclusiones 5 . Así, se puede vislumbrar que la evaluación sucede como práctica instrumental y conceptual pero no de investigación, y tampoco se dirige a las conclusiones que están directamente vinculadas con el empoderamiento de la comunidad para reflexionar sus aciertos. De este modo, la evaluación cumple una doble responsabilidad: por una parte Los programas que adoptan un proceso de evaluación empoderadora están asumiendo responsabilidad por sus propias acciones y están respondiendo, de manera confiable, ante los supervisores y agencias externas que solicitan la evaluación. Al contrario, ante las dos perspectivas: responsabilidad ante sí mismo o la responsabilidad convencional. Están tratando de transitar del enfoque de responsabilidad convencional a la función de auditoría y del enfoque de responsabilidad ante sí mismo a la función de evaluación ${ }^{7}$.

La evaluación educativa que ha tenido lugar en las IES, ha sido más instrumentalista o técnica, lo cual no es suficiente para los retos de conformarse en comunidades para el aprendizaje con impacto social en la formación de recursos humanos. Es necesario transitar del control a la responsabilidad; de la perspectiva eficientista a la de la complejidad; de la jerárquica a la democrática; de la sancionadora a la empoderadora y de la técnica a la sociopolítica ${ }^{6}$.

En la Escuela Nacional de Enfermería y Obstetricia (ENEO), específicamente, se ha vuelto la mirada a la autoevaluación como medio innovador y colaborativo interno, conducido por los propios actores 
para identificar las necesidades de la institución y realizar un diagnóstico contextualizado. Es por esta modalidad que puede constituirse una comunidad de aprendizaje reflexiva que busca la transformación a partir del diálogo, el consenso y la participación comprometida para analizar la complejidad de los fenómenos educativos en enfermería.

La ENEO cuenta con una experiencia capitalizada de más de dos décadas, para los planes de estudio, la docencia y el aprovechamiento escolar mediante exámenes estandarizados de conocimiento. La función de la evaluación es formativa, aunque no se cuenta con evidencia documentada respecto a los resultados que contribuyen a la orientación de las decisiones para la mejora y al impacto en la formación de recursos humanos de enfermería en las licenciaturas.

La dificultad más clara que enfrenta la evaluación educativa en enfermería, es evidenciar la calidad sostenida en el tiempo y en la redirección de los cambios por los actores, no sólo con datos aislados que se traducen en vacíos de significado para la comunidad que evalúa y la que es evaluada. Actualmente, en la ENEO se genera más información derivada de la evaluación educativa, que la que coadyuva, efectivamente, a la toma de decisiones por parte de la gestión académico-administrativa, los diversos cuerpos colegiados, los docentes y los alumnos.

Respecto a la autoevaluación, es el proceso de valoración de una institución educativa a partir de la información recogida de forma sistemática sobre sus fines y funcionamiento, con la finalidad de apoyar la toma de decisiones y mejorar el aprendizaje: es llevado a cabo por los propios actores ${ }^{8}$.

A diferencia del amplio desarrollo teórico en relación con la evaluación educativa, la autoevaluación tiene apenas significativas referencias de sus prácticas en las instituciones educativas. Surge con influencia de numerosos enfoques, y es una respuesta alternativa a la corriente anglosajona de la rendición de cuentas, la innovación para la mejora de la escuela, así como la del desarrollo organizacional y profesional que guarda proximidad con los modelos de evaluación participativa ${ }^{6}$. (Tabla 1).

La definición de autoevaluación se ha vinculado con las diversas denominaciones del término como son: evaluación interna, revisión interna, y evaluación basada en el centro ${ }^{6}$. Se han llevado a cabo en casi todos los sistemas educativos una variedad de experiencias donde los participantes y responsables de la organización asumen su propia evaluación?

La autoevaluación ha recibido atención del ámbito académico y cuenta en la actualidad con un cierto desarrollo teórico. Sin embargo, éste es nimio pues prevalece la práctica de autorrevisión institucional versus las definiciones oficiales de la autoevaluación en las IES, lo que ha repercutido en la diversidad de significados y modalidades.

El contexto define el término que se utiliza en cuanto a la autoevaluación, en algunos países como Estados Unidos se ve como complemento de la evaluación externa de rendimiento, en otros, se considera un sistema válido en sí mismo para evaluar un centro educativo ${ }^{10}$.

La autoevaluación se realiza en los centros educativos, por sí y para ellos mismos ${ }^{11}$. Es un proceso iniciado en el centro escolar, llevado a cabo por el profesorado con el propósito de encontrar respuestas a problemas; se orienta al diagnóstico de la situación e identificación de necesidades de una comunidad específica ${ }^{12}$. Es la mejor vía para juzgar la calidad de los procesos complejos de enseñanza-aprendizaje que tienen lugar en las IES ${ }^{13}$.

La autoevaluación se orienta en la mejora de la institución como finalidad que le da sentido ${ }^{14}$. La finalidad de la autoevaluación es el desarrollo profesional y la rendición de cuentas ${ }^{15}$. Es una herramienta para el empoderamiento y la gestión basada en el centro ${ }^{16}$, para establecer y asumir estándares de rendimiento internos y externos ${ }^{17,18}$.

La autoevaluación busca la retroalimentación con base en la construcción de significados compartidos entre los actores de la institución, posibilita el contraste de percepciones y crea vínculos de colaboración; permite construir una visión compartida sobre los beneficios y limitaciones de las prácticas de enseñanza, mediante el debate informado y provee a los actores de información periódica, válida y pertinente para desarrollar sus prácticas dirigidas a consensuar lo que hacemos y cómo deberíamos mejorarlo ${ }^{19}$. 
El impacto de los programas de innovación-evaluación en los procesos de enseñanza tiene que ver con el conocimiento y sobre la evaluación aplicada, con la interacción entre los participantes en el proceso, con el grado en que el programa de innovación-evaluación forme parte de la institución educativa ${ }^{20}$.

Algunas definiciones de autoevaluación se comentan a continuación:

\section{Tabla 1. Definiciones de autoevaluación}

Proceso de autoanálisis desarrollado por los propios integrantes de una institución, tiene su origen en la investigación de soluciones para los problemas detectados y en la búsqueda de la satisfacción de las demandas sociales ${ }^{21}$.

Comprende un área colectiva, continua y una revisión encaminada a la transformación. En este caso el énfasis se hace en el proceso, si éste es institucional e involucra a los actores que hacen posible la operación de un programa para conocer y valorar el impacto de las prácticas y los procesos que tienen sentido en la medida que logra formar a los egresados que la sociedad le demanda ${ }^{21}$.

Es un medio para identificar problemas y está orientada al cambio. Se ve enriquecida con la participación de pares externos que la complementa ${ }^{21}$.

Proceso interno para que los actores educativos reflexionen sus fortalezas y debilidades para la mejora continua ${ }^{21}$.

Proceso iniciado en el centro escolar, llevado a cabo por el profesorado y personal de la escuela, en el que se examina y diagnostica, al recoger de modo sistemático información del estado de la escuela (puntos fuertes y necesidades), con el propósito atender los problemas ${ }^{22}$

Proceso de reflexión sobre la práctica, realizado de forma sistemática y transparente, con el fin de mejorar el aprendizaje organizativo, profesional y del alumnado ${ }^{18}$.

Es la evaluación realizada por los responsables del diseño y la implementación de una intervención para el desarrollo²

Un proceso iniciado por el centro para recoger información sistemática sobre su funcionamiento, analizar y valorar esa información al tener en cuenta la calidad de los procesos educativos y tomar decisiones para realizar nuevas propuestas y recomendaciones ${ }^{24}$.

El esfuerzo colectivo de un equipo interno para someter a la organización a un examen en profundidad en base a observaciones y datos que permitan identificar sus fortalezas y debilidades, definir la evolución de la calidad de los aprendizajes de los alumnos y de gestión de la vida escolar a los cambios de la sociedad ${ }^{25}$.

Proyectos de evaluación en pequeña escala llevados a cabo por los participantes y gestores de los programas como parte de sus actividades de trabajo diario, con la finalidad de dar respuestas a cuestiones que plantea su propio trabajo ${ }^{10}$.

Proceso de recogida sistemática de información a iniciativa del propio centro y que busca valorar su funcionamiento y el logro de los objetivos educativos, con el propósito de fundamentar la toma de decisiones y contribuir a la mejora del centro en su conjunto ${ }^{8}$.

Enfoque sistemáticamente organizado y apoyado en evidencias por el que los grupos implicados revisan y supervisan regularmente el progreso y los resultados del centro, con el fin de articular iniciativas de mejora ${ }^{26}$

Proceso de reflexión, generalmente iniciado por el propio centro, que implica a los participantes en la descripción sistemática y valoración del funcionamiento del centro en orden a tomar decisiones para el desarrollo global del mismo ${ }^{27}$.

Fuente: San Fabián y Granda 20136; González-Juárez, $2010^{21}$.

Las definiciones anteriores son congruentes con la propia evolución del concepto de evaluación educativa. Se pueden extraer las siguientes características de la autoevaluación ${ }^{6}$ :

a) Proceso gestionado desde dentro que implica al conjunto de organización.

b) Se apoya en una recogida sistemática de información.

c) Implica un proceso institucional dinámico y participativo de reflexión sobre la práctica.

d) Tiene una finalidad diagnóstica de la calidad educativa.

e) Se orienta a la mejora de la organización, del personal docente y del aprendizaje del alumnado.

Con base en estos antecedentes la autoevaluación en enfermería, específicamente en las licenciaturas escolarizadas de la ENEO: la Licenciatura en Enfermería y Licenciatura en Enfermería y Obstetricia ${ }^{28}$, tuvo como objetivo impulsar la autoevaluación colegiada para identificar las fortalezas y debilidades de planes de estudio, docencia y exámenes para evaluar el aprovechamiento escolar de los alumnos.

La pregunta de investigación fue ¿cuáles son las fortalezas y debilidades de las licenciaturas en enfermería, que los actores con experiencia en evaluación educativa priorizan a través de la autoevaluación colegiada para el plan de estudios, docencia y exámenes? 


\section{Método}

El estudio fue exploratorio, con muestreo no probabilístico, después de haber analizado los perfiles de los docentes posibles se seleccionaron 27 de los 73 profesores de tiempo completo. La profesión de los participantes fue: dos psicólogos, cinco pedagogos y 20 enfermeros con experiencia en evaluación educativa en las licenciaturas escolarizadas. Se dividieron en tres equipos de acuerdo con su experiencia en evaluación: planes de estudios, docentes y alumnos. Se organizaron en grupos de nueve participantes cada uno durante 14 sesiones totales; cuatro para cada objeto de evaluación y con duración de dos horas cada una. El modelo para la autoevaluación fue Contexto, Insumo, Proceso y Producto (CIPP $)^{29}$. El modelo ha sido de amplía utilidad en educación superior con diversas perspectivas, ha servido de referencia para evaluar el currículo y programas educativos de nivel superior ${ }^{20}$.

Se diseñó un cuestionario de autoevaluación de cada objeto de evaluación con las fases del modelo CIPP. Las dimensiones y preguntas que se consideraron fueron las siguientes:

Contexto: ¿qué necesitamos hacer?, ¿podemos hacerlo?; Insumo: ¿con qué contamos? Se considera la infraestructura física, material y humana; Proceso: ¿qué o cuánto estamos haciendo?; y finalmente, Producto: ¿los resultados son los esperados y si son aceptables?

\section{Resultados}

Los 27 profesores laboraban tiempo completo en la ENEO; el 92\% fueron mujeres y 8\% hombres; 74\% de los participantes correspondían a profesionales de enfermería y $26 \%$ de otras profesiones de educación con experiencia en evaluación educativa. El diagnóstico mostró 15 fortalezas identificadas para el plan de estudios de la Licenciatura en Enfermería (Tabla 2), pues era nuevo plan en operación y se identificaron también debilidades, pues no contenía los programas de prácticas de las asignaturas ni la definición de los propios campos para realizarlas. El plan de estudios de la Licenciatura en Enfermería y Obstetricia (LEO) estaba actualizado y en operación.

Tabla 2. Fortalezas del plan de estudios de la Licenciatura en Enfermería (LE)

\begin{tabular}{lc}
\hline Fortalezas del plan de estudios & Porcentaje global (n=15) \\
\hline CONTEXTO & 26.7 \\
\hline Responde a las necesidades de salud de la población del país & \\
\hline Cumple la normativa institucional & 40.0 \\
\hline Responde a las características actuales de contratación & \\
\hline Conocimientos amplios de las diferentes disciplinas & \\
\hline INSUMO & \\
\hline Existe claridad en los objetivos & 33.3 \\
\hline Pueden cursar los semestres simultáneamente & \\
\hline Prácticas avanzadas hacia el posgrado & \\
\hline El alumno avanza a su ritmo & \\
\hline La estructura curricular es adecuada para el logro del perfil & \\
\hline RESULTADO & \\
\hline Proyección de la escuela y demanda de ingreso & \\
\hline Pueden insertarse en el campo laboral & \\
\hline Ya tienen ámbitos laborales específicos donde se tendría que observar la trascendencia \\
\hline Se espera una eficiencia terminal aceptable
\end{tabular}


Las fortalezas se orientaron al insumo y de las 22 debilidades del plan de estudios 11 (50\%) correspondieron al insumo, 4 (18.2) en contexto, 4 (18.2\%) en resultados y 3(13.6\%) en proceso. (Tabla 3).

Tabla 3. Debilidades del plan de estudios de la Licenciatura en Enfermería (LE) y Licenciatura en Enfermería y Obstetricia (LEO)

Debilidades del plan de estudios LE y LEO

Las prácticas comunitarias son relevantes y no están incluidas en el plan de estudios de la LE

Responde parcialmente en: campos de práctica, intercambio estudiantil, determinantes sociales en salud, seriación

Falta claridad entre las prácticas y su relación con la vida profesional

Desconocimiento del plan de estudios global

INSUMO

Diversificar las opciones para que sean de elección

Falta de infraestructura para un plan con un gran número de asignaturas

La estructura es buena pero muy relajada

Hay que revisar los espacios de práctica clínica (1 er nivel de atención)

Recursos humanos no preparados previamente para algunas asignaturas

Poca claridad en relación con las asignaturas

Buscar optativas de elección

Hace falta elaborar los programas de prácticas clínicas y comunitarias que guíen la formación

Falta formar más a los docentes del CECA

Mejorar la organización de los laboratorios de enfermería para que los alumnos puedan utilizarlos

Es muy importante preparar docentes para la práctica comunitaria

PROCESO

Más herramientas de autoevaluación para compensar carencias para LE y LEO

Falta realizar acciones en función del proyecto de desarrollo de los programas de práctica

No hay estrategias de práctica análoga

RESULTADOS

No se cuenta con estudios de egresados

Falta planear mejor la práctica del CECA

Mejorar la práctica clínica para tener egresados bien preparados (LE y LEO)

El egresado tiene formación básica y amplia, pero requiere más práctica (LEO)

En el caso de evaluación de la docencia, se identificaron 5 fortalezas (Tabla 4) y 9 debilidades (Tabla 5). En el primer caso, principalmente, se ubican en el insumo y dos del contexto, mientras que las debilidades se focalizan en los resultados de bajo impacto de esta estrategia institucional.

Tabla 4. Fortalezas de evaluación de la docencia en enfermería

Fortalezas de evaluación de la docencia

Porcentaje

global $(n=5)$

CONTEXTO

40.00

Pertenencia de evaluar la práctica docente

Perfil docente basado en conocimientos, habilidades y actitudes

INSUMO

Contar con un perfil de docencia para la licenciatura en las dimensiones de clínica, laboratorio y aula

Congruencia entre la evaluación docente y el modelo de docencia en la ENEO que se puede proponer

Mecanismos de evaluación dirigidos a mejorar la práctica docente 
Tabla 5. Debilidades de evaluación de la docencia en enfermería

Debilidades de la evaluación de la docencia

La evaluación de la docencia en la eficacia de los aprendizajes de los alumnos

Evaluación de pares para fortalecer la evaluación de la docencia

\begin{tabular}{ll}
\hline INSUMO & 11.11 \\
\hline Contar con instrumentos válidos y confiables para evaluar la docencia & 22.22 \\
\hline PROCESO & 33.3 \\
\hline Evaluar la docencia sólo mediante la opinión de los estudiantes \\
\hline RESULTADOS docencia centrada en el logro de objetivos de aprendizaje \\
\hline Afinar procedimientos y tiempos para evaluar la docencia
\end{tabular}

Generar información de la evaluación de la docencia contribuye a la toma de decisiones para la mejora

La evaluación de la docencia es poco congruente con los criterios de calidad de la licenciatura en la ENEO

Evaluación de poco impacto en la mejora de las prácticas de los docentes

En el caso de evaluación mediante exámenes muestran cinco fortalezas en total consensuadas por los participantes, las de mayor porcentaje se refieren al proceso (Tabla 6) e identificar las diferentes trayectorias de los alumnos a partir de los aprendizajes evaluados.

Tabla 6. Fortalezas de la evaluación de los alumnos mediante exámenes Fortalezas de la evaluación de los alumnos: exámenes

\begin{tabular}{lc}
\hline CONTEXTO & 20.0 \\
\hline Participación de los profesores de las academias para elaborar los reactivos de las pruebas & 20.0 \\
\hline INSUMO & 40.0 \\
\hline Presencia de exámenes de avance y colegiados & \\
\hline PROCESO & 20.0 \\
\hline Evaluar aprendizajes mediante el avance en la formación disciplinar de los alumnos & \\
\hline Identificar las diferencias en las trayectorias de los alumnos a partir de los aprendizajes evaluados & \\
\hline PRODUCTO & \\
\hline Algunas academias cuentan con información de la evaluación de los aprendizajes
\end{tabular}

Las debilidades de la evaluación de los alumnos mediante exámenes fueron 10 en total (Tabla 7). De las cuales $60 \%$ se ubica en los resultados de bajo impacto para tomar decisiones de mejora, para lo que se refiere a los exámenes colegiados y el EPAC, requiere integrar otros conocimientos adicionales a los cognitivos.

Las dificultades institucionales se identificaron en los planes de estudios, especialmente, en el nuevo de Licenciatura en Enfermería que carecía de los programas de práctica, ya que su orientación es teórico-práctica. Las mayores debilidades se ubicaron en el bajo impacto de los exámenes colegiados para valorar el aprovechamiento escolar de los estudiantes del sistema escolarizado. Esto último cobra relevancia para la evaluación formativa comprometida en el currículo. 
Tabla 7. Debilidades de la evaluación de los alumnos mediante exámenes

\begin{tabular}{lc}
\hline Debilidades de la evaluación de los alumnos: exámenes & Porcentaje global (n=10) \\
\hline INSUMO & 20.0 \\
\hline No se asegura la pertinencia de los contenidos de la prueba & 20.0 \\
\hline Vigencia de los contenidos declarativos de la prueba & \\
\hline PROCESO & 60.0 \\
\hline Equidad de participación de los actores de la comunidad en la aplicación de las pruebas & \\
\hline Autoevaluación colegiada de los resultados de la prueba & \\
\hline RESULTADOS & \\
\hline Asegurar que las pruebas respondan a perfiles de conocimiento, habilidad y actitud & \\
\hline La evaluación de aprendizajes orientada a la toma de decisiones de mejora en la comunidad & \\
\hline Valorar el impacto institucional de evaluar el aprendizaje \\
mediante exámenes en las trayectorias escolares
\end{tabular}

\section{Discusión}

La pregunta que guío el estudio fue identificar las fortalezas y debilidades de los planes de estudio, la evaluación de la docencia y los exámenes colegiados para el fortalecer el aprovechamiento escolar de los alumnos de las licenciaturas escolarizadas en enfermería de la ENEO.

La autoevaluación permitió obtener un diagnóstico de los componentes descritos con ejercicio pionero que favoreció la reflexión, aunque no se establecieron estándares de rendimiento internos y externos referidos en otros estudios ${ }^{17}$, dado el carácter indagatorio del estudio.

La evaluación educativa realizada en las Instituciones de Educación Superior, generalmente la realizan para cumplir con las acreditaciones nacionales, de tal suerte que la autoevaluación educativa se convierte en una gestión administrativa burocratizada ${ }^{5}$, esto representa resistencias de los actores para la reflexión interna del cambio que favorece el empoderamiento de la comunidad que la realiza. En este estudio, no se identificaron resultados de impacto de la evaluación realizada en las licenciaturas para los componentes descritos en realidad, el trabajo colegiado que implicó mirarse a sí mismos permitió identificar las áreas más fuertes y débiles en los componentes analizados.

La estructura especifica de cuerpos colegiados dirigidos a revisar y analizar la información recuperada contribuyó no sólo a la reflexión de la comunidad para la mejora, sino también permitió identificar las sinergias necesarias para impulsar el cambio y a los actores responsables de éste. Asimismo, sirvió como guía para el seguimiento en el tiempo.

Una limitación importante del estudio son los participantes, la autoevaluación fue impulsada como una estrategia institucional, y los criterios de inclusión de la muestra fueron la experiencia y el conocimiento experto en materia de evaluación educativa ${ }^{10,11}$, no obstante, si se busca conformar una comunidad de aprendizaje, reflexiva ${ }^{7}$ e incluyente, se hace necesaria la participación de todos los actores de la escuela.

La autoevaluación colegiada se orientó en la mejora y no así en la calidad educativa ${ }^{13}$, noción necesaria en próximos ejercicios para juzgar los procesos complejos de enseñanza-aprendizaje, que tienen lugar en la ENEO y que se configuran como parte de la responsabilidad social de esta actividad en la Universidad. El trabajo colegiado y estratégico fue la forma de atender las acciones de mejora ${ }^{14},{ }^{19}$ para informar a los órganos colegiados de la escuela. 


\section{Conclusiones}

La autoevaluación institucional ha sido una modalidad poco referida en la institución. La validez de la información da soporte a la evaluación educativa que se realiza, por ello, resulta relevante realizarla por fases y de forma colegiada, participativa.

La evaluación en enfermería ha seguido la tradición centrada en la opinión del alumno para valorar a los docentes, perspectiva unilateral y limitada para tomar decisiones de mejora en los componentes analizados. También, se basa en la tradición cognitiva del aprendizaje, para valorar los conocimientos de los alumnos mediante exámenes que se realizan por los docentes que participan en las doce academias.

En el caso de la evaluación por exámenes colegiados para mejorar el aprovechamiento de los alumnos, no permitió identificar las decisiones de mejora que toman los docentes, a partir de los resultados obtenidos en sus grupos; lo cual, es una debilidad de la evaluación formativa en la ENEO, pues se carece de un estudio respecto a las decisiones que los docentes y los alumnos toman para superar el riesgo de abandono o reprobación durante el semestre lectivo. Se conocen los riesgos que implica la evaluación centrada en un sólo actor, en tanto es una forma de alteración entre la teoría y la práctica que pone en riesgo la validez de la evaluación que se realiza, por lo tanto es poco justa para integrar a los actores y su contexto.

Finalmente, aunque el modelo teórico y metodológico de base para llevar a cabo la autoevaluación, permitió estructurarla en el contexto de enfermería, es importante consensuar algunas definiciones con todos los participantes para mejorar el trabajo colegiado al iniciar el ejercicio, además considerar que la presencia de un experto en evaluación contribuye a que los grupos operen de forma eficiente y focalizada.

\section{Responsabilidades éticas}

Protección de personas y animales. Los autores declaran que para esta investigación no se han realizado experimentos en seres humanos ni en animales.

Confidencialidad de los datos. Los autores declaran que han seguido los protocolos de su centro de trabajo sobre la publicación de datos de pacientes.

Derecho a la privacidad y consentimiento informado. Los autores declaran que en este artículo no aparecen datos de los participantes.

Financiamiento. Ninguno.

Conflicto de intereses. No hay ningún conflicto de intereses.

\section{Referencias}

1. Torres-Santomé J. La justicia curricular. El caballo de Troya de la cultura escolar. Madrid: Morata S.L.; 2011.

2. García-Cabrero B. Modelos teóricos e indicadores de evaluación educativa. Sinéctica. 2010;(35). [Consultado abril 12 2018]. Disponible en: https://bit.ly/2EfS22e

3. González JG. La evaluación educativa en ciencias en la salud: un esbozo del marco de la responsabilidad social universitaria. CDMX: ENEO UNAM; (en edición).

4. Martínez-Rizo F. La evaluación formativa del aprendizaje en el aula en la bibliografía en inglés y francés. Rev. Mex. Inv. Educ. 2012; 17(54): 849-75. [Consultado abril 10 2018]. Disponible en: https://bit.ly/2G05xop

5. Weiss CH. Utilization of evaluation: Toward comparative study. En: Weiss CH. Evaluating action programs: Readings in Social Action and Education. Boston: Allyn \& Bacon; 1972.

6. San Fabián-Maroto JL, Granda-Cabrales A. Autoevaluación de centros educativos. Cómo mejorar desde dentro. Madrid: Síntesis; 2013.

7. Fetterman D. Empowerment Evaluation: su aplicación en la educación universitaria. En: Congreso RedLatEM. México: Facmed UNAM; 2018. [Consultado 5 junio 2018]. Disponible en: 
https://bit.ly/2E08Qtn

8. Schildkamp K. The utilisation of a self-evaluation instrument. Educational Studies. 2010; 36(4): 371-89. https://doi.org/10.1080/03055690903424741

9. Scheerens J. School self-evaluation: Origins, definition, approaches, methods and implementation. In: Nevo D. School-based evaluation: An international perspective. Oxford: Elsevier Science; 2002. p. 35-69.

10. Taut S. Studying self-evaluation capacity building in a large international development organization. Am J Eval. 2007; 28(1): 45-59. http://journals.sagepub.com/doi/10.1177/1098214006296430

11. Swaffield S, MacBeath J. School self-evaluation and the role of a critical friend. Cam J Ed. 2005; 35(2): 239-52. [Consultado junio 13 2018]. Disponible en: https://bit.ly/2QyLPEC

12. Bolívar-Botía A. Los centros educativos como organizaciones que aprenden. Promesas y realidades. Madrid: Muralla; 2000.

13. Janssens FJG, Van Amelsvoort GHWCH. School self-evaluations and school inspections in Europe: An exploratory study. Studies in Educational Evaluation. 2008; 34(1):15-23. https://doi.org/10.1016/j.stueduc.2008.01.002

14. Vanhoof J, Van Petegem P. Feedback of performance indicators as a strategic instrument for school improvement. REICE. 2005; 3(1): 206-21. [Consultado abril 9 2018]. Disponible en: https://bit.ly/2E5wGnp

15. Simons H. School self-evaluation in a democracy. In: Nevo D. School-based evaluation: An international perspective. Oxford: Elsevier Sciencie; 2002.

16. Nevo D. Dialogue evaluation: Combining internal and external evaluation. En: Nevo D. School-based evaluation: An international perspective. Oxford: Elsevier Science; 2002.

17. Rallis SF, MacMullen MM. Inquiry-minded schools: Opening doors for accountability. Illinois: Phi Delta Kappan; 200

18. MacBeath J. Schools must speak for themselves. The case for school self-evaluation. London: Routledge; 1999.

19. MacBeath J. School Self-evaluation: Background, Principles and Key Learning. Nottingham: National College for School Leadership; 2005.

20. Murray R. Citizens' Control of Evaluations. Formulating and Assessing Alternatives. Evaluation. 2002; 8(1): 81-100. [Consultado mayo 19 2018]. Disponible en: https://bit.ly/2QDqBFs

21. González-Juárez G. Autoevaluación Institucional: Retos para la mejora de los programas de posgrado de la UNAM. En: Barrón C, Valenzuela A. El Posgrado. Programas y actores. CDMX: IISUE-UNAM; 2013.

22. Bolívar A. Autoevaluación institucional para la mejora interna. En: Zabalza MA. Reforma educativa y organización escolar. Santiago de Compostela: Tórculo; 1994.

23. Organisation For Economic Co-Operation And Development. Evaluation feedback for effective learning and accountability. Paris: OECD; 2001. [Consultado 19 mayo 2018]. Disponible en: https://bit.ly/2snyTG4

24. Devos G, Verhoeven JC. School Self-Evaluation-Conditions and Caveats: The Case of Secondary Schools. Educ. Manag. Adm.Leadersh. 2003; 31 (4): 403-20. [Consultado 25 mayo 2018]. Disponible en: http://doi.org/10.1177/0263211X030314005

25. Bonami M. Évaluation interne et évaluation externe : concurrence ou Complémentarité. HAL archives-ouvertes. 2005; (38): 1-20. [Consultado 25 mayo 2018]. Disponible en:

https://bit.ly/2BUpo4D

26. Plowright D. Self-evaluation and Ofsted Inspection. Developing an Integrative Model of School Improvement. Educ. Manag. Adm. Leadersh. 2007; 35(3): 373-393.

https://doi.org/10.1177/1741143207078180

27. Van-Petegem P, DeneireA, De Maeyer S. Evaluation and participation in secondary education: Designing 
and validating a self-evaluation instrument for teachers to solicit feedback from pupils. Studies in Educational Evaluation. 2008; 34(3):136-44. https://doi.org/10.1177/1741143207078180

28. Universidad Nacional Autónoma de México-Escuela Nacional de Enfermería y Obstetricia. Licenciatura en Enfermería-Licenciatura en enfermería y obstetricia. Generalidades, objetivos y quehacer profesional. CDMX: UNAM-ENEO [Consultado junio 3 2018]. Disponible en: https://bit.ly/2FUH1Vf

29. Stufflebeam DL. The CIPP model for program evaluation. En: Stufflebeam DL, Madaus GF, Kallaghan T. Evaluation models. Switzerland: Springer Netherlands; 1983. 